\section{Hybrid Surgery for Extraction of Leads Entrapped in the Superior Vena Cava}

Superior vena cava (SVC) syndrome is an uncommon but serious complication associated, among other things, with chronic transvenous implanted pacemaker leads. (1) Although there is lack of consensus about how to treat this complication, percutaneous balloon angioplasty of the SVC with stent implant is a commonly accepted approach leaving the leads in situ, or another option is prior lead extraction followed by their reimplantation after the procedure to avoid lead entrapment within the stent. Epicardial lead implantation could reduce the risk of thrombosis associated with transvenous reimplantation. (2)

Hybrid open heart surgery and transvenous lead extraction using sheaths has developed considerably over the past several decades. Although transvenous lead extraction is the standard procedure to remove infected or malfunctioning leads, a surgical approach may be necessary in complex cases that involve concomitant conditions, such as tricuspid valve regurgitation or luminal lead entrapment. (3)

We report the case of a patient with pacemaker leads entrapped in a stent previously implanted in the SVC to treat occlusive syndrome due to SVC thrombosis, who underwent hybrid cardiac surgery with simultaneous transvenous lead removal.

The patient was a 65-year-old man with history of dual chamber pacemaker implanted in 2007 due to complete atrioventricular block. Six months later, a passive fixation lead was percutaneously implanted via the left subclavian vein due to failure of the ventricular active fixation lead, which was left abandoned. In 2014, the pacemaker generator was changed due to battery exhaustion.

In 2017 the patient presented SVC syndrome an underwent angioplasty of the SVC with placement of a $20 \times 80 \mathrm{~mm}$ self-expanding nitinol Sinus-XL stent (Opti Mcd®), with its distal part left projecting towards the right atrium. After the implant, the SVC syndrome improved, and symptoms reverted. By the end of 2018, the patient presented ventricular pacing failure due to intracavitary lead fracture. After confirming that the three intracavitary leads in the SVC were entrapped in the stent and could not be removed, an epicardial ventricular lead was inserted through a left thoracotomy and was placed over the left ventricle. One month later, the patient presented pocket site infection with external exposure of the device, negative blood cultures and no evidence of endocarditis.

Because of infection and stent entrapment of three leads in the SVC, the entire stimulation system was removed from a pacemaker-dependent patient. A cavography was performed to analyze stent patency (Figure 1) and the patient underwent a multislice computed tomography (CT) scan to plan the strategy for surgical extraction (Figure 2). A combined procedure for lead extraction was decided: open surgery by median sternotomy, with cardiopulmonary bypass and aortic cross clamping, and a percutaneous approach through the left pocket where the generator was implanted. The right atrium was incised, and the stent was identified inside. The three intracavitary leads were released from the right ventricle and right atrium until reaching the stent in the SVC. Simultaneously, the three leads were percutane- ously released from the fibrous tissue in the left subclavian and innominate region until reaching the proximal edge of the stent. Stylet wires, a liberator and $10 \mathrm{Fr}$ polypropylene dilator sheaths (Cook $\left.{ }^{\circledR}\right)$ were used. Two leads were sectioned above the stent and removed through the atrium, and the third lead was extracted through the pocket. The epicardial catheter was sectioned at the level of the pericardium and removed through the pocket.

Aortic cross-clamp time was 45 minutes and cardiopulmonary bypass time was 82 minutes. Epicardial leads were placed for temporary pacing and the patient was treated with antibiotics. One week after lead extraction, two intracavitary leads were implanted through the stent and the postoperative course was uneventful.

Percutaneous extraction is the technique of choice for removing leads from infected cardiac stimulation devices with low rates of major complications and mortality. (4) A recent systematic review including more than 3,000 patients reported an average success rate of $92.4 \%$ for complete percutaneous removal of infected leads. The incidence of major complications and minor complications was $2.9 \%$ and $8.4 \%$, respectively. In-hospital mortality was $5.4 \%$ and procedurerelated mortality ranged from $0.4 \%$ to $3.6 \%$. Mean mortality rate was $20 \%$ at 6 months and $14 \%$ at one-year follow-up. (5)

There is only one case report of successful lead extraction of infected pacing leads trapped by a stent in a patient with surgically corrected transposition of the great arteries. In this case, manual traction was sufficient to remove the leads. (6) Another case of entrapment in the innominate vein corresponded to an ossified thrombus 18 years after pacemaker implantation; on that occasion, the lead was extracted using a laser sheath. (7) As with the patient here

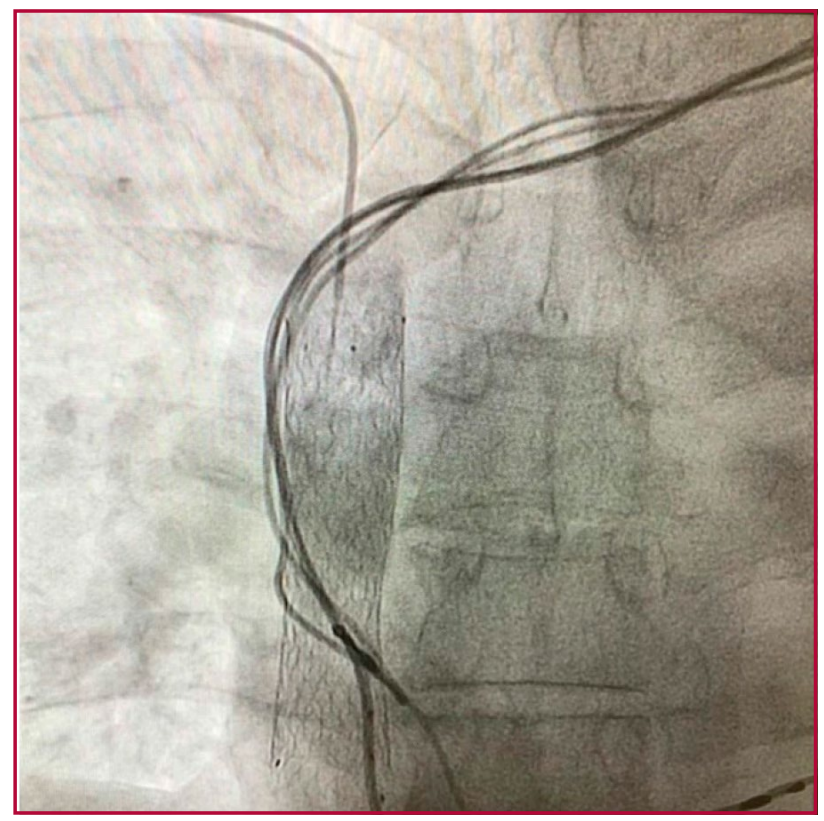

Fig. 1. Detail of the cavography, showing the different pacing leads and a patent stent in the superior vena cava. 


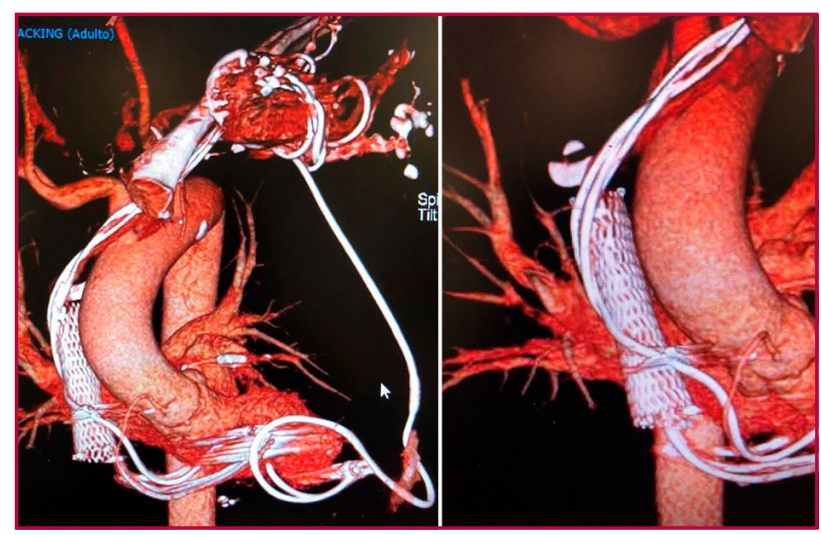

Fig. 2. Three-dimensional reconstruction of the multislice computed tomography scan, showing the different pacing leads and the stent implanted in the superior vena cava (right image: detail of the entrapped leads).

presented, compression of pacemaker leads by a stent in the SVC is exceptional and constitutes a challenge for extraction. Hybrid cardiac surgery with simultaneous percutaneous extraction was a safe option to treat this unusual and complex case. In view of these results, the complexity of extracting leads entrapped between a stent and the SVC wall should be considered in case of SVC syndrome requiring stent placement.

\section{Conflicts of interest}

None declared.

(See authors' conflicts of interest forms on the website/ Supplementary material).

Ethical considerations

$\mathrm{RAB}$ is Director of the Argentine Journal of Cardiology.

Félix Ramírez, Héctor A. Mazzetti, Arnaldo Milani, Raúl A. Borracci (i) Department of Cardiovascular Surgery, Clínica y Maternidad Suizo Argentina. Av. Pueyrredón 1461, C1015, Buenos Aires, Argentina E-mail: FelixRi@swissmedical.com.ar

\section{REFERENCES}

1. Zucchelli G, Favilli E, Viani S, Barletta V, Di Cori A, Segreti L, et al. Leadless pacing in a patient with superior vena cava syndrome undergoing lead extraction and percutaneous angioplasty. J Cardiol Cases 2018;17:212-4. https://10.1016/j.jccase.2018.02.008.

2. Maseda Uriza R, Jurado-Román A, Jimenez Díaz J, Piqueras Flores J, Higuera Sobrino F, Oliva De Anquín E. Hybrid Approach for the Treatment of Superior Vena Cava Syndrome Induced by Pacemaker. Ann Thorac Surg 2017;104:e131-e132. https://10.1016/j. athoracsur.2017.02.063.

3. Azarrafiy R, Carrillo RG. Surgical and Hybrid Lead Extraction. Card Electrophysiol Clin 2018;10:659-665. https://10.1016/j. ccep.2018.07.006.

4. Menezes Júnior ADS, Magalhães TR, Morais AOA. Percutaneous Lead Extraction in Infection of Cardiac Implantable Electronic Devices: a Systematic Review. Braz J Cardiovasc Surg 2018;33:194-202. https://10.21470/1678-9741-2017-0144.

5. Bongiorni MG, Burri H, Deharo JC, Starck C, Kennergren C, Saghy L, et al; ESC Scientific Document Group. 2018 EHRA expert consensus statement on lead extraction: recommendations on definitions, endpoints, research trial design, and data collection requirements for clinical scientific studies and registries: endorsed by APHRS/HRS/ LAHRS. Europace 2018;20:1217. https://10.1093/ europace/euy050.

6. Shetty AK, Walker F, Cullen S, Lambiase PD.Extraction of pacing leads jailed by a stent in a mustard circulation. Pacing Clin Electrophysiol 2010;33:e65-7. https://10.1111/j.1540-8159.2010.02710.x. 7. Okamura H, Van Arnam JS, Aubry MC, Friedman PA, Cha YM. Successful pacemaker lead extraction involving an ossified thrombus: A case report. J Arrhythm 2017;33:150-1. https://10.1016/j. joa.2016.06.007.

Rev Argent Cardiol 2019;87:359-360.

http://dx.doi.org/10.7775/rac.v88.i4.16019

\section{Complete Left Bundle Branch Block and Blunt Cardiac Injury: A Lesson Learned}

Chest injury is the second leading cause of mortality after head injury, and accounts for $20-25 \%$ of all accidental deaths. (1) Even though the treatment of patients with polytrauma exceeds cardiological management, patients with closed chest trauma presenting with arrhythmias, elevated cardiac enzymes or pain may require our evaluation. These findings may indicate blunt cardiac injury, a condition in our specialty that causes high mortality. Most cardiac complications secondary to blunt cardiac injury due to closed chest trauma occur within the first 24 hours (65\% are already present on admission), (2) and cardiologists should be alert to their clinical presentation and outcome.

We report the case of a 26 -year-old male patient with no previous relevant history who was admitted after falling from a motorcycle due to frontal collision with a car. He presented polytrauma with closed-chest and right forearm trauma, encephalocranial trauma and posterior loss of consciousness. On admission, the electrocardiogram (ECG) showed complete atrioventricular block (AVB), QRS complex with complete right bundle branch block (RBBB) morphology and heat rate (HR) of $25 \mathrm{bpm}$ (Fig. 1a). On physical examination, the patient presented an open forearm fracture (Gustilo I) and appeared lucid without signs suggestive of cerebral ischemia and with normal blood pressure. Some minutes later, 3:1 AVB with LBBB conduction developed that was not present on an ECG taken the previous year. After isoproterenol administration, the HR increased to $60 \mathrm{bpm}$ (Fig. 1b). An echocardiogram was urgently performed, with normal results. The laboratory tests showed elevated troponin I level of $2.16 \mathrm{IU} / \mathrm{L}$ (normal value $<0.02 \mathrm{IU} / \mathrm{L}$ ). The patient presented complete AVB and complete LBBB during the first 24 hours after admission and was asymptomatic; then, the heart rhythm alternated between sinus rhythm and sinus arrest with persistent complete LBBB. The tests performed to evaluate polytrauma did not show costal fractures, but the computed tomography scan showed signs of pulmonary contusion, right pleural effusion and distal radi- 